\title{
El maxilar inferior del Orden Lagomorpha como material de investigación en Implantología Experimental
}

\author{
DE CORRAL ESCOBAR A * \\ MESA AGUADO FL ** \\ FREIHERR VON FORSTNER V *** \\ O'VALLE RAVASSA FJ ****
}

\begin{abstract}
De Corral Escobar A, Mesa Aguado FL, Freiherr von Forstner V, O'Valle Ravassa FJ. El maxilar inferior del Orden Lagomorpha como material de investigación en Implantología Experimental. Av Periodon Implantol. 2002; 14, 2: 57-62.
\end{abstract}

\begin{abstract}
RESUMEN
El propósito de este trabajo es presentar un nuevo modelo experimental para el estudio de implantes en la mandíbula del conejo por la cara inferior o región milohioidea, donde se encuentra un tejido óseo en forma triangular, constituido por hueso compacto en superficie y trabecular en profundidad, semejante al del tejido alveolar del maxilar humano, que puede ser de interés como una nueva localización para estudiar la osteointegración de implantes. Se utilizaron 12 conejos europeos machos de 2.5 a $3 \mathrm{Kg}$, se colocaron un total de 12 implantes Dentatus ${ }^{\oplus}$ TRl de titanio de $8 \mathrm{~mm}$ y se realizó el estudio histológico con técnicas histoquímicas y tratamiento de imagen por ordenador.
\end{abstract}

\section{PALĀBRĀS CLAVE}

Implante dental, osteointegración, conejo, modelo experimental.

\section{INTRODUCCIÓN}

Las investigaciones de implantes dentales sobre animales han sido realizadas en diferentes mamíferos como el Mono Macacus rhesus (1), Macaca especiosa (2) y fascicularis (3), diversas razas de perros ("beagel" y labrador) (4-6) y el cerdo $(7,8)$, todos ellos tienen inconvenientes de su manipulación por su tamaño, volumen e idiosincrasia, crean problemas de estabulación y son modelos animales caros para manejo en gran número.

Si bien el aparato estomatognástico tanto de los órdenes de Primates y Carnívora, como el suborden Bunodontia (cerdo) tienen similitudes anatomo-fisiológicas con el humano y todos ellos poseen diaste- mas, lo que permite la fijación de implantes y estudian la osteointegración bajo diferentes condiciones de carga (8), técnicas quirúrgicas (9), evaluación de los cambios óseos tras administración de alendronatos (10) y estudios histológicos $(5,11)$. El alto costo de los Primates así como su cuidadosa manutención hizo que muchas investigaciones se derivaran a perros y más recientemente a cerdos, ya que al ser omnívoros tienen un tipo de trituración en la masticación similar a los movimientos del maxilar humano. Por otra parte la longitud de los dientes es comparable a los del hombre y es relativamente resistente a las infecciones por lo tanto conveniente para la cirugía bucal (12).

Cuando se precisa realizar un estudio estadístico con gran número de animales es conveniente recurrir a

* Licenciado en Odontología. Práctica Privada.

** Médico Estomatólogo. Prof. Asociado de Periodoncia. Facultad de Odontología. Universidad Granada.

*** Doctor en Veterinaria. Universidad de Munich.

**** Profesor Titular de Anatomía Patológica. Facultad de Medicina. Universidad Granada. 
modelos de experimentación fáciles de manipular y mantener. Por esta razón nosotros recurrimos al Oryctolagus cuniculus L. (conejo europeo).

De los animales de laboratorio, el conejo es un animal dócil, fácil de estabular (13), es relativamente simple de anestesiar y tiene bajo costo de alimentación lo que lo hace útil en investigación en implantología experimental. Presenta una estructura ósea semejante a la humana; pero el género Oryctolagus presenta el inconveniente de poseer un esqueleto sumamente ligero, ya que supone solamente el $7 \%$ del peso, cantidad ciertamente reducida si se la compara con el $21.5 \%$ del cerdo o el $25 \%$ en el hombre. El conejo ha sido profusamente empleado para la investigación de implantes óseos, fundamentalmente empleando la diáfisis o los cóndilos tibiales (14-21). En la mandíbula del conejo por la borde inferior o milohioideo, tras realizar cortes seriados orientados transversalmente, hemos observado un tejido óseo con morfología triangular, constituida por hueso compacto y trabecular en profundidad, semejante al del tejido alveolar humano que puede constituirse como una nueva localización para estudiar la osteointegración de implantes.

\section{MATERIAL Y MÉTODOS}

Se utilizaron 12 conejos europeos machos de 2.5 a $3 \mathrm{Kg}$ de peso suministrados por el animalario de la Universidad de Granada. Los animales fueron estabulados individualmente con alimentación vegetal y agua ad libitum. Para la manipulación y sacrificio de los animales se siguieron las normativas y directivas vigentes del Consejo de Europa (86/609/CEE, RD 223/1988).

Se desarrolló una técnica anestésico-quirúrgica adaptada a nuestro modelo consistente en:

Anestesiar a los animales con asociación sinérgica de ketamina (Imalgine ${ }^{\circledast 500}$ ) a dosis de $0.1 \mathrm{ml} / \mathrm{Kg}$ de peso y xylacina al $2 \%$ (Xilagesic $^{\oplus} 2 \%$ ) a dosis 0.2 $\mathrm{ml} / \mathrm{Kg}$ vía intramuscular en el glúteo del animal. Este sinergismo produce en el género Oryctolagus una somnolencia, que va acompañada de amplia analgesia cuya profundidad depende de la dosis y no se interrumpe por la acción de estímulos externos. El tiempo de anestesia básica fue de 8 minutos, que se prolongó durante 40 minutos con posterior estado de somnolencia de 1 hora.

El abordaje quirúrgico fue en la región milohioidea en el borde inferior de la mandíbula sobre la bifurcación de su cuerpo con la rama montante. El procedimiento quirúrgico constó de las siguientes fases:

Se rasuró la región submandibular del animal y se realizó una incisión en "Y" griega invertida procediéndose a la diéresis de los tejidos, teniendo particular precaución de no lesionar la arteria y vena faciales que cabalgan sobre el borde inferior de la mandíbula.

Se perforaron la dos cuñas óseas con una fresa quirúrgica número 151 de $23 \mathrm{~mm}$ de longitud (Maillefer) siguiendo el eje que debe coincidir con la cúspide del $2^{\circ}$ premolar o primer molar y su punto de inserción de 1.5 a $2 \mathrm{~mm}$ por delante del primer premolar inferior.

Se colocó en el hueso un implante Dentatus ${ }^{\circledast}$ TRl de titanio de $8 \mathrm{~mm}$ de longitud total (Dentatus $A B$, Hägersten, Suiza) y se realizó la sutura de los tejidos y la limpieza de la herida con Aluspray ${ }^{\odot}$ (Vetoquinol, Cedex, Francia), partículas de aluminio en aerosol que por astringencia favorece la cicatrización. La mandíbula contralateral, utilizada como control, se dejó sin implante.

Tras la intervención se hizo necesaria la inyección subcutánea de 10-20 ml, (en ocasiones hasta $40 \mathrm{ml}$ ) de suero glucosalino al 10\% en distintas partes del cuerpo del animal para evitar su muerte debido a la hipoglucemia que provocan la mezcla de anestésicos empleados.

Los maxilares fueron disecados y fijados en formalina tamponada al $10 \%$. Un lote de ellos se incluyó en metacrilato de metilo + peróxido de benzoilo y otro lote se decalcificó con ácido etilendiaminotetracético (EDTA, Sigma Che. Chershire, UK) y se incluyó en parafina. Se realizaron coloraciones de hematoxilinaeosina de Wallington, tricrómico del Goldner y azul de toluidina $(22,23)$.

\section{RESULTADOS}

De los 12 conejos empleados, los 3 primeros murieron en el período postquirúrgico inmediato por hipoglucemia al no suministrarles suero glucosalino. En los 9 restantes no se produjeron complicaciones derivadas de la intervención ni sufrieron infecciones durante el período de mantenimiento hasta su sacrificio. Los animales fueron implantados en un tiempo máximo de 30 minutos y no se perdió ningún implante por manipulación de los animales o por defecto en su colocación. Los implantes fueron colocados con una angulación variable que osciló entre $70^{\circ}$ para el ángulo alfa y $110^{\circ}$ para el ángulo beta (figura 1). En el momento del sacrificio tras 11 semanas, una vez disecada la mandíbula, se valoró la movilidad de los implantes comprobándose macroscópicamente la correcta osteointegración.

El estudio histológico del área de implantación expe- 

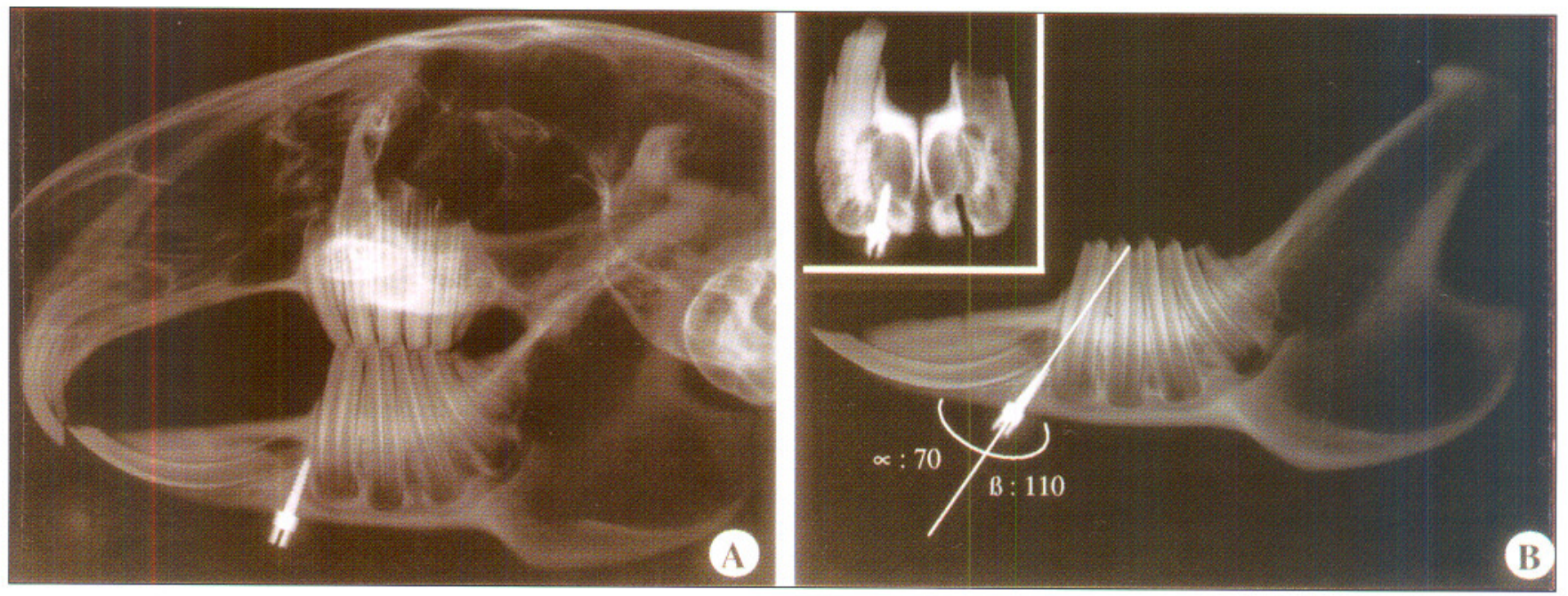

Fig. 1: Radiografías laterales de cráneos de conejo europeo realizadas a distancia focal de $50 \mathrm{~cm}, 70 \mathrm{Kw}, 15 \mathrm{~mA}$ y 0.40 segundos de exposición. A) Situación del implante una vez colocado en la cuña ósea submaxilar elegida para el modelo experimental. B) Orientación del eje del implante con las amplitudes más correctas de los ángulos alfa y beta. Inserto:Visión anteroposterior de un corte de maxilar inferior implantado. Nótese las variaciones en las angulaciones que del implante en diferentes animales.

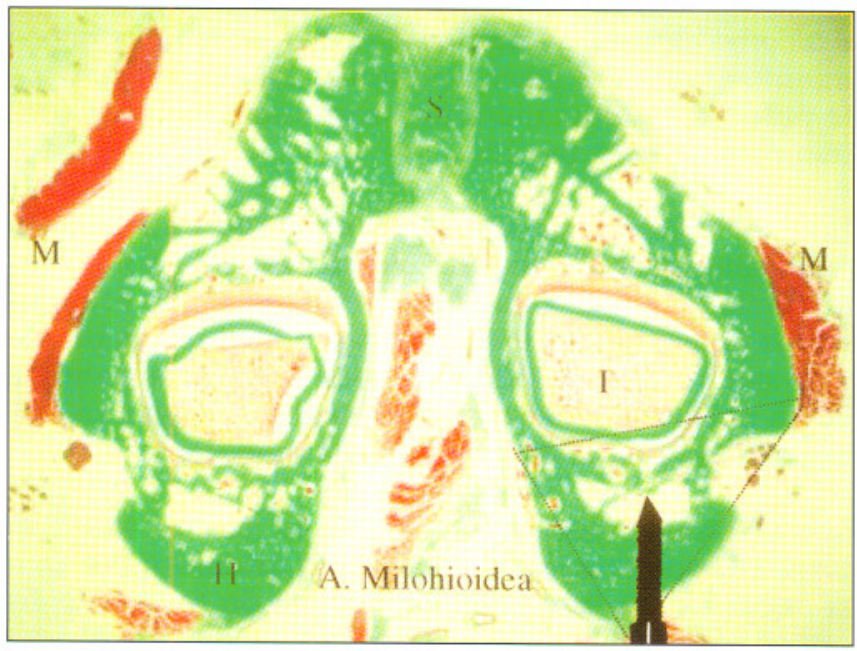

Fig. 2: Corte transversal de maxilar inferior a la altura del germen dentario del incisivo de crecimiento contínuo donde esquemáticamente se ha señalado la posición del área de implantación y la disposición del implante. H: tejido óseo, M: tejido muscular, S: sínfisis mentoniana, I: incisivo de crecimiento contínuo. (Tricómico de Goldner $5 x$ ).

rimental mostró un tejido óseo compacto, fasciculado con pocas cavidades medulares y de aspecto maduro, que se extendía entre el borde inferior del maxilar y el incisivo de crecimiento continuo. El área elegida tiene forma triangular y fue estimada sus dimensiones mediante un portaobjetos milimetrado en $6 \pm 0.05$ $\mathrm{mm}^{2}$ para conejos de $2.5 \mathrm{Kg}$ con leves variaciones dependiendo del tamaño del animal. Los sistemas de Havers fueron más pequeños que los humanos con un diámetro medio de $100 \mu \mathrm{m}$, rodeado generalmente de cuatro capas de laminillas y un conducto de Havers de $14 \mu \mathrm{m}$ de diámetro, con 123 conductos por $\mathrm{mm}^{2}$ frente

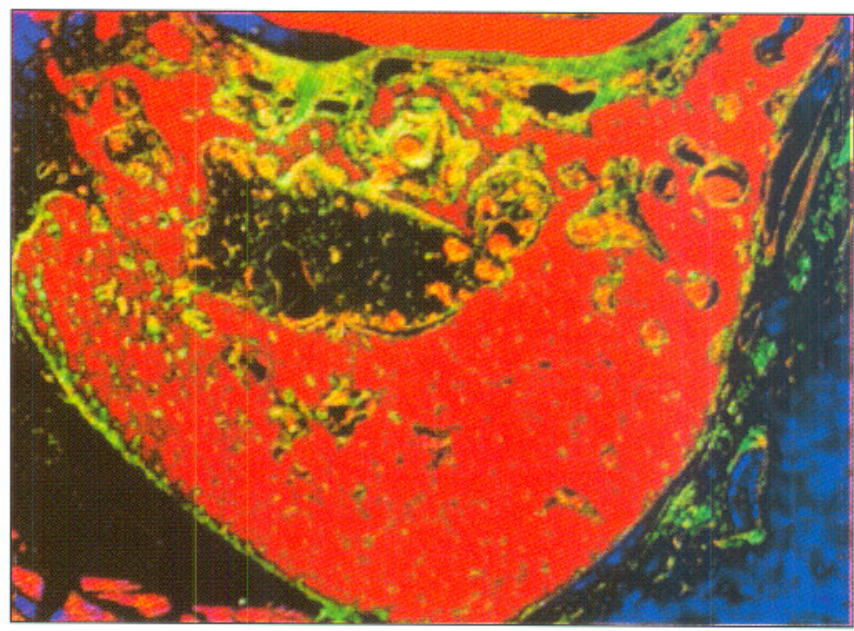

Fig. 3: Zona de implantación submaxilar a mayor aumento y tras tratamiento con análisis de imagen por ordenador. Se aprecia hueso Haversiano compacto con intenso color rojo rodeado de periostio que destaca de color amarillo (Tricrómico de Goldner 40x).

a 80.6 conductos por $\mathrm{mm}^{2}$ que se encuentran en la diáfisis tibial.

Los cortes decalcificados teñidos con tricrómico de Goldner adoptaron una tinción verde al unirse el colorante verde luz al tejido óseo compacto maduro. La madurez ósea se comprobó histológica e histoquímicamente ya que ningún conducto de Havers se tiñó con el colorante orange $\mathbf{G}$ (figura 2). El tratamiento informático de las imágenes permitió reconocer en la zona de implantación el área perióstica que adoptó color amarillo y el hueso compacto de color rojo homogéneo (figura 3).

En la figura 2 se detallan las relaciones anatómicas de 
la zona de implantación mandibular y en la figura 1 en una radiografía anteroposterior se de una sección de la mandíbula, puede apreciarse la situación y aspecto final de la colocación de los implantes.

\section{DISCUSIÓN}

El establecimiento de un nueva localización para implantes óseos requiere de un conocimiento previo de su anatomía e histología, necesarios para poder interpretar los cambios producidos y el pronóstico de los implantes, por ello y al no existir ningún trabajo metodológico sobre este modelo experimental hemos llevado a cabo este estudio.

El conejo tiene diferentes lugares a priori adecuados para la colocación de implantes. El primero y más utilizado es la tibia (14-21). La segunda zona de implantación experimental podría ser intrabucal, como se emplea en animales superiores y en tercer lugar el borde inferior de la mandíbula, en la zona milohioidea, que constituye el modelo experimental desarrollado en este trabajo.

En la tibia los lugares de fijación son los $2 / 3$ inferiores de la diáfisis por carecer de inserciones musculares (14-21), si las investigaciones se quieren hacer en hueso haversiano o en los cóndilos si se quiere estudiar específicamente hueso compacto y esponjoso (21). El abordaje quirúrgico se hace separando los músculos bíceps femoral y gastrocnemio en un primer plano, y los músculos extensor dígito largos y laterales en un segundo plano quirúrgico. Las ventajas además de su fácil acceso quirúrgico es la posibilidad de colocar múltiples implantes en cada animal. Presenta un inconveniente importante, lo reducido del espesor del hueso compacto diafisario (espesor medio $0.66 \pm 0.04 \mathrm{~mm}$ en el conejo europeo), lo que requiere implantes de pequeña longitud, que aun así penetran en la zona medular, la cual presenta una escasa estructura ósea trabecular.

La implantación intrabucal tendría la ventaja de poder extrapolar los resultados a los implantes humanos ya que las condiciones son aproximadas a las del hombre (cavidad bucal séptica, trauma masticatorio y presencia de restos alimenticios). Un gran diastema entre los incisivos inferiores y primer premolar permitiría la fijación de implantes; Pero técnicamente las desventajas son muchas: la apertura bucal en el conejo es reducida debiéndose usar instrumentos adecuados (abrebocas y separadores de A. Albrecht ${ }^{\circledR}$ ), actualmente no se dispone de instrumental quirúrgico específico con dimensiones tan reducidas, el espesor de hueso en el diastema es menor, el tamaño del implante debe ser más reducido, etc.
La zona milohioidea escogida para este modelo, presenta tejido óseo en forma triangular muy vascularizado cuyo vértice interno es el final de la sutura de tipo sincondrosis de la parte incisiva del cuerpo mandibular (figura 2). El tejido óseo es más homogéneo y la parte interna constituida por hueso trabecular es semejante al del tejido alveolar humano (24). A diferencia del hombre los sistemas de Havers de la mandíbula parecen ser iguales a los de otros huesos de la economía como la tibia (25). Es de gran importancia el hallazgo de hueso haversiano en la cuña ósea que describimos, porque aunque este tipo de tejido es propio de primates superiores, no es el único modelo, ni es el más frecuente, existiendo una gran variedad histológica en la estructura ósea entre animales de diferente taxonomía, entre huesos diferentes del mismo animal y en relación también con la edad del animal (26). No se ha encontrado hueso acelular patrimonio de otros roedores como las ratas donde el número de osteones es más bien escaso con amplias zonas de hueso acelular. Este hueso considerado más ancestral (25) es muy común en vertebrados inferiores como anfibios y reptiles (27), que presentan crecimiento estacional, pero también se halla en importante proporción en mamíferos como marsupiales, insectívoros y carnívoros (28).

El error técnico más frecuente consistió en atravesar con el implante el germen dentario del incisivo de crecimiento continuo. Este hecho ocurrió cuando el ángulo alfa constituido por el eje longitudinal del implante y la rama horizontal de la mandíbula fue superior a $90^{\circ}$. A pesar de haber ocurrido en dos animales no evidenciamos alteración en el crecimiento dental.

Los implantes dentales empleados fueron tornillos torneados de titanio sin tratamiento de superficie, que se recubren de una capa de dióxido de titanio $\left(\mathrm{O}_{2} \mathrm{Ti}\right)$ por su oxidación con el aire, de modo que el contacto se estable entre este dióxido de titanio y el tejido receptor (29), incrementándose el anclaje del implante con la maduración ósea durante la reparación (30). Por las reducidas dimensiones del modelo, los tornillos empleados como implantes corresponde al material para la realización de la reconstrucción de coronas en operatoria dental. No obstante, el material que lo constituye es similar al de los implantes usados en clínica humana.

El diámetro, la composición, el tratamiento de la superficie del implante e incluso la forma tienen repercusión sobre los resultados de la osteointegración del mismo. Recientemente, diferentes autores han demostrado que tratamientos con hidroxiapatita, fosfato cálcico y materiales cristalinos (45S5) mejoran los resultados (31). Gotfredsen y colaboradores demostraron mayor densidad ósea alrededor de implantes tratados en superficie con plasma que en los de $\mathrm{O}_{2} \mathrm{Ti}$ sólo 
torneados y que existe una clara relación entre la rugosidad de la superficie, determinada por el grosor de las partículas de revestimiento de Ti (de 10 a 125 $\mu \mathrm{m})$ y el anclaje durante la osteointegración (30). Hayakawa comprobó la mejor respuesta ósea a los implantes revestidos con fosfato tricálcico (32).

Nuestro método permite el uso de un grupo de animales numeroso y homogéneo que se adapta al estudio histomorfométrico de parámetros estáticos y dinámicos de la respuesta ósea al implante. La aplicación de marcadores fluorescentes como alizarina roja $\mathrm{S}$, el xilenol naranja, la dimetilclorotetraciclina o la calceina azul y la cuantificación mediante microscopia láser confocal nos permitirá estudiar la remodelación ósea y los fenómenos de osteointegración en la interfase hueso-implante en el modelo propuesto.

\section{AGRADECIMIENTOS}

Los autores queremos manifestar nuestro agradecimiento a la Sra. Judith Gómez Villaescusa, técnico de laboratorio por la realización de las técnica histológicas.

\section{ABSTRACT}

The aim of this paper was to show a new experimental model for the study of rabbit jaw implants on the lower face or mylohyoid region, where we founded bone tissue with triangular shape compound for compact bone tissue in surface and trabecular bone in depth, similar to human alveolar tissue of jaw, that should be interesting as a new location for implants osseointegration studies. We used 12 male European rabbits of 2.5 a $3 \mathrm{Kg}$ weigh, were put a total of 12 titanium implants Dentatus TRl of $8 \mathrm{~mm}$ long and hitological study with histochemical techniques and computer image treatment were done.

\section{KEY WORDS}

Dental Implant, osseointegration, rabbit, experimental model.

\section{CORRESPONDENCIA}

Francisco Luis Mesa Aguado Avda. Medina Olmos $\mathrm{n}^{\circ} 22, \mathrm{l}^{\circ} \mathrm{B}$ 18500 Guadix-Granada. España
Telf./fax: 958663132

e-mail: fmesa@ugr.es

\section{BIBLIOGRAFÍA}

1. Busser, D.; Warren, K. and Karting, Th. Titanium implants with a true periodontal ligament: An alternative to osseointegrated implants? Oral Maxillofacial Im. 1990, 5: 113116.

2. Schroeder, A.; Van der Zypen, E.; Stich, H. et al. The reactions of Bone Connective Tissue and epithelium endosteal implants with titanium sprayed surfaces. J. Maxillofaci. Surg. 1981, 9: 11-25.

3. ISIDOR, F. Clinical probing and radiographic assessment in relation to the histologic bone level at oral implant monkeys. Clin. Oral Implant. Res. 1997, 8: 255-264.

4. Gotfredsen, K.; Berglundh, T.; Lindhe, J. Bone reactions adjacent to titanium implants subjected to static load. A study in the dog (I). Clin. Oral Implants Res. 2001, 12:1-8.

5. Persson, L.G.; Ericsson, I.; Berglundh, T. and Lindhe, J. Osseointegration following treatment of peri-implantitis and replacement of implant components. An experimental study in the dog. J. Clin. Periodontol. 2001, 28: 258-263.

6. Barbier, L.; Vanden Sloten, J.; Krzesinski, E. et al. Fine element analysis of non-axial versus axial loading of oral implants in the mandible of the dog. J. Oral Rehabilitation. 1998, 25: 847-858.

7. Karagianes, M.T.; Westerman, R.E.; Rasmussen, J.J. et al. Development an evaluation of porous ceramic and titanium alloy dental anchors implanted in miniature swine. J. Biomed. Mater. Res. 1974, 5: 391-399.

8. Hale, T. M.; Boretsky, B.B.; Scheidt, M.J. et al. Evaluations of titanium dental implant osseointegration in posterior edentulous areas of micro swine. J. Oral Implantol. 1991, 17: 118-124.

9. Bråneman, P.I. Osseointegration and its experimental background. J Prosthet. Dent. 1983, 50: 399-410.

10. Meraw, S.J. and Reeve, Ch. M. Qualitative analysis of peripheral peri-implant bone and influence of alendronate sodium on early bone regeneration. J. Periodontol. 1999, 70: 1228-1233.

11. Hipp, J.A.; Brunski, J.B. and Cochran, G.V.B. Method for histological preparation of bone section containing titanium implants. Stain Tech. 1987, 62: 247-252.

12. Kalkwarf, K.L.; Krejci, R.F.; Edison, A.R. and Reinhardt, 
R.A. Sujacent heat production during excicion with electrosurgery. J. Oral Maxillofac. Surg. 1983, 41: 653-657.

13. Harrness, J.E. and Wagner, J.E. Biología y clínica de conejos y roedores. Editorial Acribia. Zaragoza. 1980.

14. Albrektsson, T. Fibrin adhesive system (FAS) influence on the bone healing rate. A microradiographical evaluation using the bone growth chamber. Acta Orthop. Scand. 1982, 53: 757-763.

15. Albrektsson, T. and Linder, L. Bone injury caused by curing bone cement. An in vivo study in the rabbit tibia. Clin. Orthop. 1984, 183: 280-283.

16. Albrektsson, T.; Hansson, H.A. and Ivarsson, B. Biomaterials. London. Butterworth Ltd. 1985.

17. Turunen, T.; Peltola, J.; Helenius, H. et al. Bioactive glass and calcium carbonate granules as filler material around titanium and bioactive glass implants in the medullar space of the rabbit tibia. Clin. Oral Impl. Res. 1997, 8: $96-$ 102.

18. Ivanoff, C.J.; Sennerby,L.; Johansson, C. et al. Influence of implant diameters on the integration of screw implants. An experimental study in rabbits. Int. J. Oral Maxillofac. Surg. 1997, 26: 141-148.

19. Ivanoff, C.J.; Sennerby, L. and Lekholm, U. Influence of soft tissue contamination on the integration of titanium implants. An experimental study in rabbits. Clin Oral Impl. Res. 1996, 7: 128-132.

20. Köning, B.; Beck, T.J.; Kappert, H.F. et al. A study of different calcification areas in newly formed bone 8 weeks after insertion of dental implant in rabbit tibias. Anat. Anz. 1998, 180: 471-475.

21. Ivanoff, C.J.; Sennerby, L. And Lekholm, J. Influence of initial implant movility on the integration of titanium implants. An experimental study in rabbits. Clin Oral Implant. 1996, 7: 120-127.

22. Bancroft, J. and Stevens, A. Theory and practice of histological techniques. Churchill Livingstone, London 1990.
23. García Del Moral, R. Laboratorio de anatomía patológica. Interamaericana·McGraw-Hill, Madrid 1993.

24. Johnson, L.C. Morphologic analysis in pathology: The kinetics of disease and general biology of bone. En: Harold M. Bone biodynamics. Frost Ed. Little Brown and Company, 1964, p 585.

25. Smith, M.M. and Hall, B.K. Development and evolutionary origins of vertebrate skeletogenic and odontogenic tissues. Biol. Rev. 1990, 65: 277-373.

26. Wainwright, S.A.; Biggs, W.D.; Currex, J.D. and Gosline, J.M. Diseño mecánico en organismos. H. Blume ediciones Madrid, 1980.

27. Francillon, H. And Castanet, J. Mice en evidence experimentale du caractere annuel des lignes d'arret de croissance squeletique chez rana esculenta (amphibia anura). C.R. Sci III, 1985, 8:327-332.

28. Kardong, K.V.Vertebrados; anatomía comparada, función evolución. $2^{\circ}$ ed, 1999 Interamericana McGraw-Hill, Madrid.

29. Kasemo, B. Biocompatibility of titanium implants surface science aspects. J. Prosthet. Dent. 1983, 49: 832.

30. Gotfredsen K, Berglundh T, Lindhe J. Anchorage of titanium implants with different surface characteristics: an experimental study in rabbits. Clin. Implant Dent. Relat. 2000, 2: 120-128.

31.Wheeler, D.L.; Montfort, M.J. and McLoughlin S.W. Differential healing response of bone adjacent to implants coated with hydroxyapatite and 45S5 bioactive glass. J. Biomed. Mater. Res. 2001; 15: 603-612.

32. Hayakawa, T.; Yoshinari, M.; Nemoto, K.; Wolke, J.G. and Jansen, J. Effect of surface roughness and calcium phosphate coating on the implant/bone response. Clin. Oral Implants Res. 2000, 11:296-304. 